\title{
Side and position of haemodialysis catheters (VasCath) can affect its survival
}

\author{
Eid Hussien, SAS Anaesthetics, South Tyneside NHS Foundation Trust. \\ Riccardo Scano, Consultant Anaesthetist, South Tyneside NHS Foundation Trust.
}

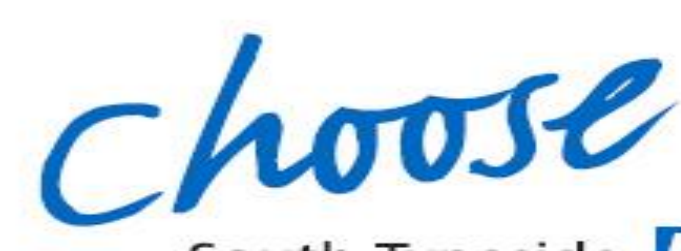

\section{Why this audit? \\ - Reduce cost of haemodialysis including catheters (VasCath): one kit costs 500 f (1). \\ - Reduce incidence of therapy interruption, risks due to malfunctioned catheters. \\ - Achieve a target of 1:1 catheter to episode of AKI (up to 7 days).}

\section{Aims and Objectives}

- To establish the site and tip position of VasCath that most likely survives and works effectively during the whole period of continuous dialysis.

\section{Specific criteria/standards}

- Adult CVC checklist and infection control procedures to be completed for $100 \%$ of VasCath inserted (trust's policy).

- Ultrasound should be used routinely for internal jugular central venous catheter insertion and of a recommended use for all other central venous access sites with limited evidence $(2,3)$.

- Whether preference of the RT side IJV/Sub VasCath insertion over the Lt side IJV/Sub leads to a longer survival rate of the catheter or not? (no strong evidence).

- Temporary haemodialysis catheter tip should be positioned in the superior vena cava/ RT atrium (at the level of the carina in CXR).

\section{Methodology}

A retrospective systematic review of all ITU patients' records over the period of 6 months from 1st of November 2015 to the 1st of May 2016, and selecting patients who had been haemodialysed through a VasCath at any point.
- Total number of patients $=115$

- Patients who received haemodialysis=25

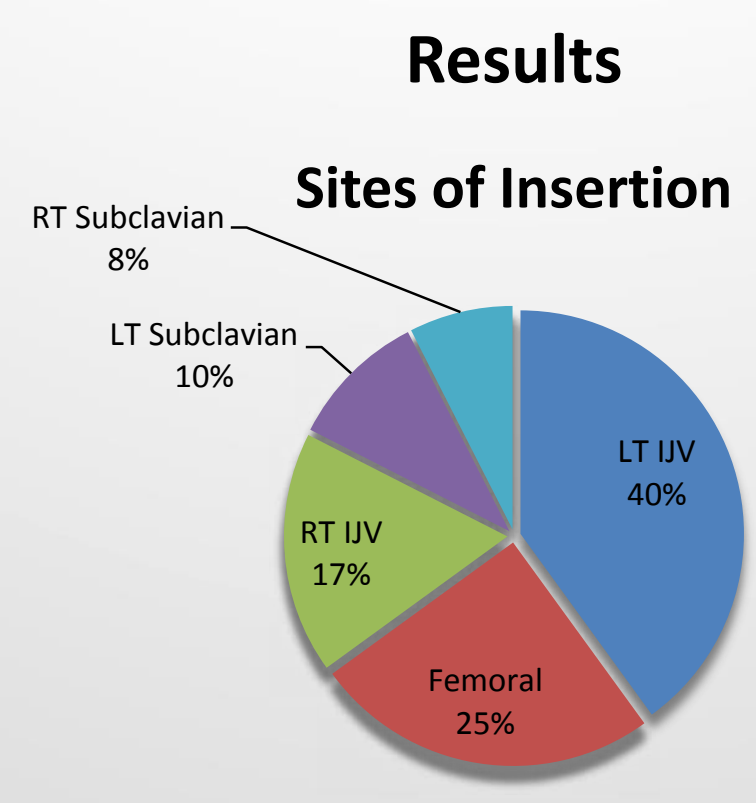

Survival in hours
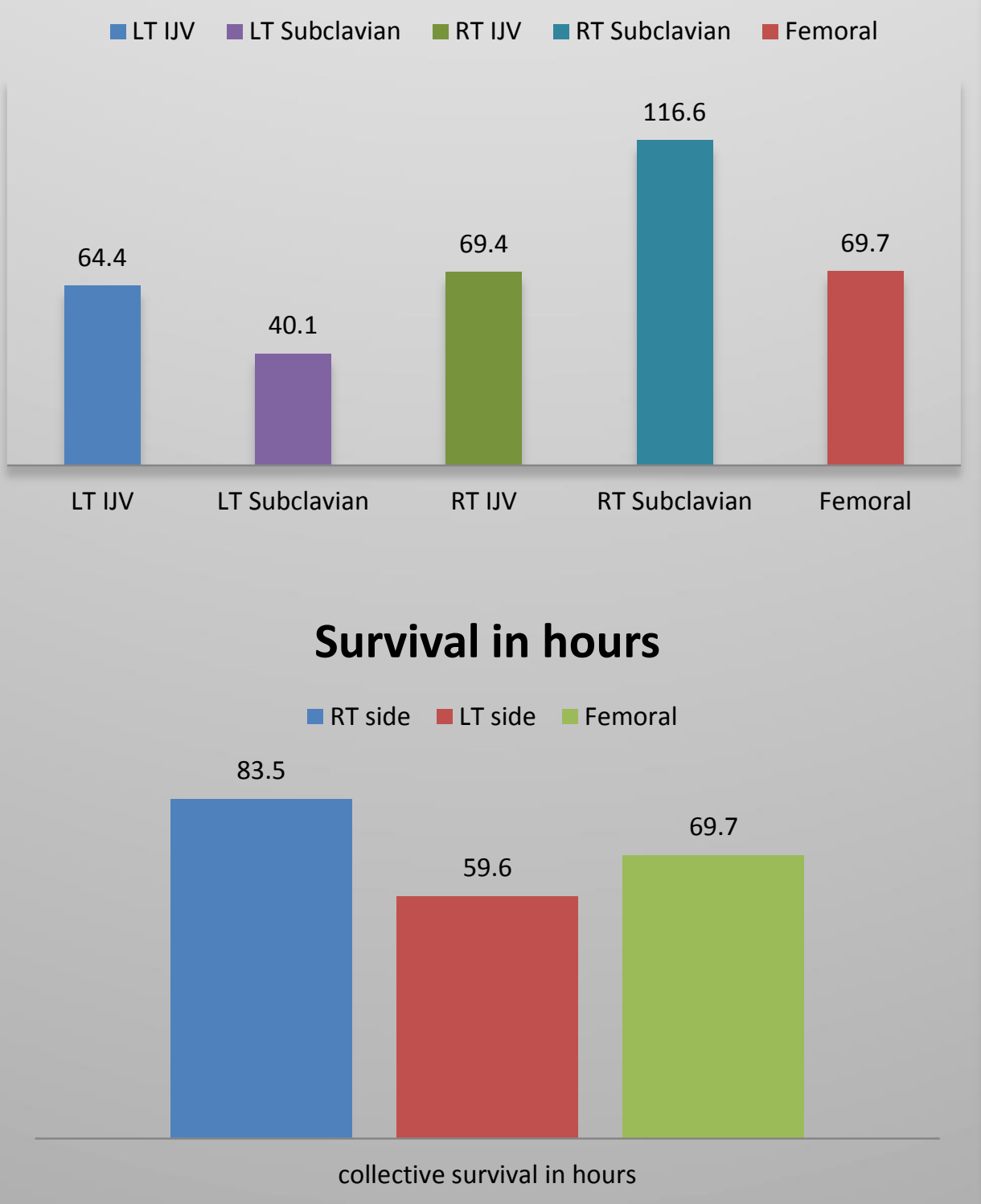

- Survival of RT side chest catheters was significantly longer than those on LT chest side and femoral $(P<0.01)$.

How often do we need to swap the lines, reposition or rewire the dialysis catheters?

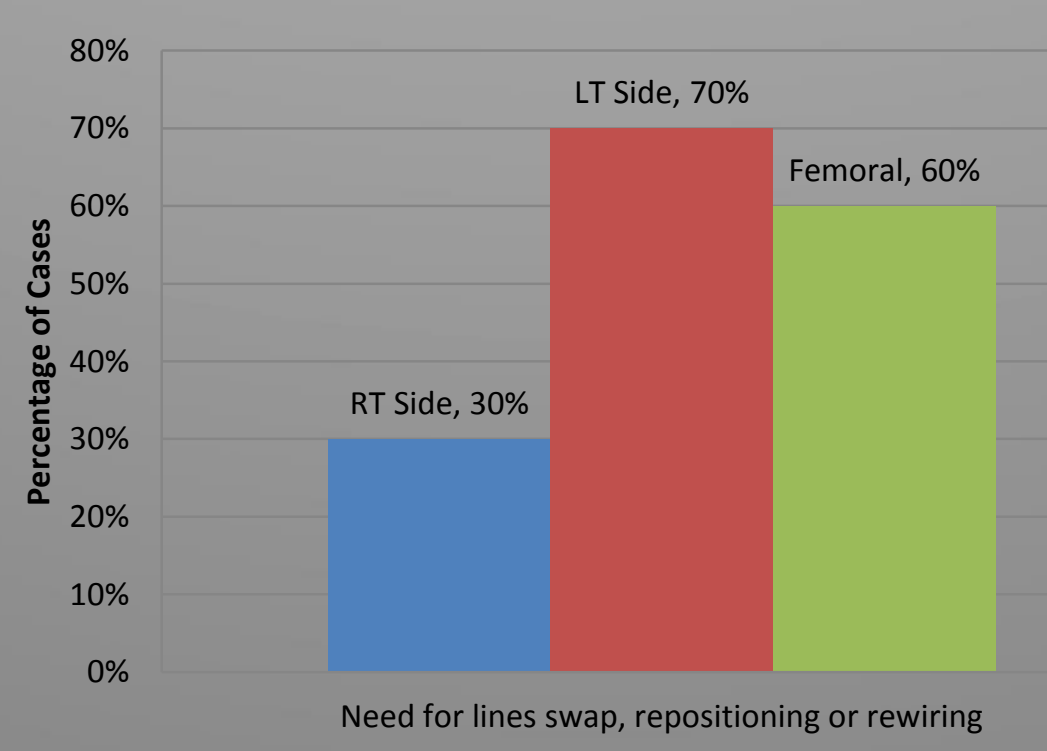

\section{Frequency of tip position}

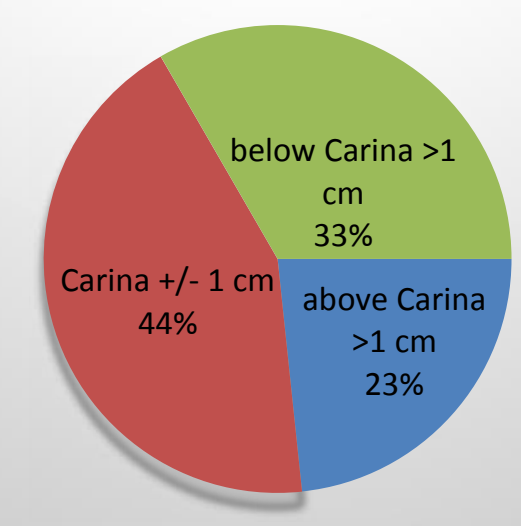

Average Survival in Hours According to Tip Position
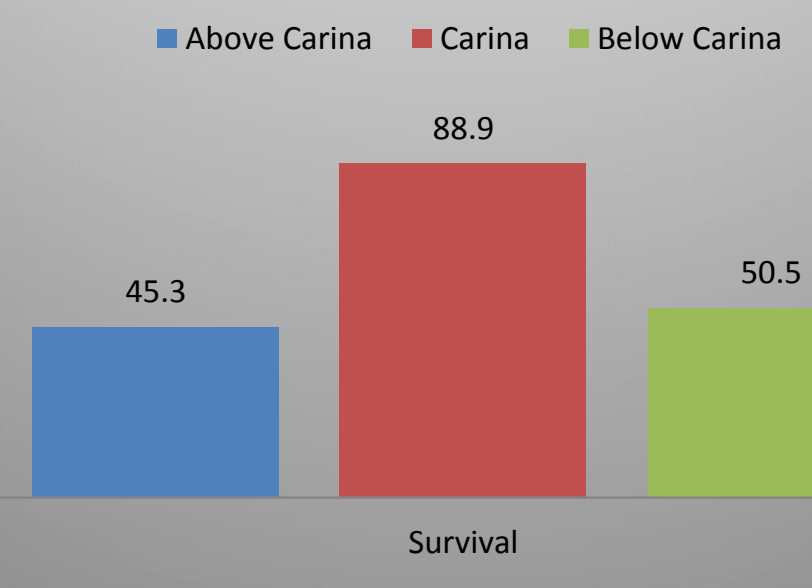

Discussion and recommendations for change

- There was a statistically significant longer survival rate for the right chest side and femoral haemodialysis catheters than left side and catheter tip position at the level of the carina $(-/+) 1 \mathrm{~cm}$ over far deep or superficial catheter tips.

- Fewer number of interruptions of dialysis therapy were encountered in right side chest catheters in comparison to left side and femoral catheters.

- favour left side CVC insertion in any patient with AKI and/or potential need for dialysis to spare right side or using the same vein dual catheter technique to obtain a vascular access and VasCath in the same vein on the right side.

\section{Action Plan}

- Highlight the value of future plan of vascular access for every ITU admission.

- Re-audit the dialysis catheters over a longer period of time with larger sample size.

- Explore all options to create a multicentre RCT to prove or deny the superiority of right side catheters over the left side.

References

1) Cochrane Database of Systematic Reviews 2011, Issue 11. Art. No.:CD005279, Published by John Wiley \& Sons, Ltd. http://www.cochranelibrary.com/cochrane-database-of-systematicreviews/table-of-contents/2011//ssue1, accessed on 15/06/2017.

2) NICE Guidance 2002 on the use of ultrasound locating devices for placing central venous catheters https://www.nice.org.uk/guidance/ta49. Accessed on 15/06/2017.

3) AAGBI Safe Vascular Access Guidelines, May, 2016 https://www.aagbi.org/publications/guidelines/safe-vascular- 